\title{
Structural and physicochemical characteristics of One- Step PAMAM dendrimeric nanoparticles
}

3 Sergio Andrés Torres-Pérez ${ }^{1}$, Luis Vallejo-Castillo ${ }^{2,8}$, Said Vázquez-Leyva ${ }^{2,8}$

4 Luis Gerardo Zepeda-Vallejo ${ }^{3}$, José Enrique Herbert-Pucheta ${ }^{3,4}$, Childérick

5 Severac ${ }^{6}$, Etienne Dague ${ }^{5}$ Sonia Mayra Pérez-Tapia ${ }^{2,7,8}$, Eva Ramón-Gallegos ${ }^{1^{*}}$

${ }^{1}$ Laboratorio de Citopatología Ambiental, Departamento de Morfología, Escuela Nacional de Ciencias Biológicas, Instituto Politécnico Nacional, Campus Zacatenco, Mexico City, Mexico.

2Unidad de Desarrollo e Investigación en Bioprocesos (UDIBI), Escuela Nacional de Ciencias Biológicas, Instituto Politécnico Nacional, Mexico City, Mexico.

${ }^{3}$ Departamendo de Química Orgánica, Escuela Nacional de Ciencias Biológicas, Instituto Politécnico Nacional, Mexico City, México

${ }^{4}$ Consejo Nacional de Ciencia y Tecnología-Laboratorio Nacional de Investigación y Servicio Agroalimentario Forestal, Universidad Autónoma Chapingo, Carretera México-Texcoco Km 38.5, 56230 Chapingo, Estado de México, México.

${ }^{5}$ LAAS, Université de Toulouse, CNRS, 31400 Toulouse, France.

${ }^{6}$ ITAV-CNRS, Université de Toulouse, CNRS, 31106 Toulouse, France.

${ }^{7}$ Departamento de Inmunología, Escuela Nacional de Ciencias Biológicas, Instituto Politécnico Nacional. Mexico City, Mexico.

${ }^{8}$ Laboratorio Nacional para Servicios Especializados de Investigación, Desarrollo e Innovación (I+D+i) para Farmoquímicos y Biotecnológicos (LANSEIDI-FarBiotec-CONACyT). Escuela Nacional de Ciencias Biológicas, Instituto Politécnico Nacional. Mexico City, Mexico.

Correspondence: Eva Ramón-Gallegos*

Environmental Cytopathology Lab, Department of Morphology, Escuela Nacional de Ciencias

Biológicas, Instituto Politécnico Nacional, Campus Zacatenco, Calle Wilfrido Massieu Esquina Cda.

Manuel Stampa, 07738, Mexico City, Mexico.

Tel: +52(55) 57296300 Ext. 52400 and 52399.

Fax: +52(55) 57296300 Ext. 52400

Email: eramong@ipn.mx 


\section{Abstract}

The recently developed One-Step Poly(amidoamine) (OS-PAMAM) dendrimers

46 stand out for their characteristics as the high drug-load capacity and cell-delivery

47 improvement of therapeutic agents. The OS-PAMAM dendrimers have proven to be useful in the biomedical field as nanocarrier or nanosystems for therapy. In the 49 present research it was encouraging to determine their physicochemical 50 characteristics which were compared with commercial PAMAM generation-6 (G6).

51 The spectroscopic measurement of amides, nanoparticle size (10-30 nm), 52 polydispersity index and zeta potential measurements correlates with the 53 commercial product. The OS-PAMAM has cavities detected by AFM, and the force

54 analysis showed the same adhesion force and different elasticity than PAMAM-G6.

55 The molecular weight (MW) was 10 times lower than the commercial one for both techniques employed, resembling the MW of a PAMAM generation-3 (G3). OSPAMAM/PAMAM-G6 MS-MS mirror plots demonstrate chemical equivalency amongst herein analyzed dendrimers, with the advantage that OS-PAMAM is produced with a faster and low-cost synthetic protocol, which allows them to be applied for both research and industry in the biomedical field.

61 Keywords: Poly(amidoamine), Nanotechnology, Molecular Weight, PFG-DOSY

62 NMR, orthogonal polydispersity index. 
73 Polyamidoamine (PAMAM) dendrimers are organic nanoparticles (NPs) that have occupied a prominent position in research and industry because of their welldescribed characteristics, high molecular loading ability, and their wide range of applicability in fields like biomedicine for controlled drug delivery, gene therapy, theragnostic and vaccines [1,2].

PAMAM dendrimers have an arborescent construction and present a generational growth form, which is based on a nucleus initiator called zero generation (G0), from which are added functional groups in the periphery resulting in homo-structural plates between focal points (branching points). Thus, a dendrimer with six branches from the centre to the periphery is called a dendrimer of generation $6(\mathrm{G} 6)[3,4]$.

Since the pioneer method of synthesis reported by Tomalia et al. 1986 [3], various strategies have been proposed for improving the PAMAM dendrimers synthesis, including divergent and/or convergent synthesis alongside click chemistry and Microwave-Assisted Reactions [5-7]. The divergent synthesis consists of a multifunctional initiator core that grows to the periphery through two reactions where dendrimers can reach generation 10. However, structural defects are a critical concern for high generation dendrimers. They inevitably arise from incomplete reactions or side reactions that occur because of the increasing number of reactions and steric hindrance as the dendrimer grows. Additionally, it is challenging to remove defective structural molecules because of their similar chemical composition [8].

On the other hand, in the convergent synthesis, dendrimers are built from small molecules starting from the surface of the dendrimer to end up attached to a central core through several inward-directed reactions. Otherwise, it can be so much controlled, and the impurities can be separated more easily, but the reactivity to reach the interior core is often considerably reduced because of the increasing steric congestion as the dendrimer generation increases. Therefore, only low-generation dendrimers can be successfully prepared using the convergent method $[4,9]$. A combined divergent/convergent method has been further developed and applied for dendrimer synthesis. This approach includes the synthesis of building blocks using 
102

103

104

105

106

107

108

109

110

111

112

113

114

115

116

117

118

119

120

121

122

123

124

125

126

127

128

129

130

131

a divergent approach followed by the convergent dendrimer assembly [8], also optimized by click chemistry and Microwave-Assisted to deliver simplified and accelerated dendrimer synthesis [6,10]. Nevertheless, the obtention of high generation PAMAM dendrimers in the desired quantity, with consistent quality and low cost and energy expenditure, remains challenging [5].

The recently developed OS-PAMAM dendrimers obtained by a novel synthesis method remain the beneficial characteristics such as high load capacity and the increased efficiency in drug delivery — like the commercial one- previously reported [11]. Moreover, the time, cost, and energy of manufacturing are reduced, enabling their massive production and application as platforms for several molecules in the biomedical field and others [12]. In this work, the novel synthesis method was employed, and the physicochemical characteristics of OS-PAMAM were compared with a commercial PAMAM G6. Hence, the characteristic functional groups expected for PAMAM dendrimers were assigned from ultraviolet-visible spectrophotometry (UV-Vis) and Fourier-Transform Infrared spectroscopy (FT-IR). Particle size distribution as a function of polydispersity indexes (PDI) as well as zeta potential (ZP) were measured by dynamic light scattering (DLS). Polydispersity in solution of both OS-PAMAM and PAMAM G6 was monitored with pulsed-field gradient saturation transfer echo water-presaturation diffusion ordered spectroscopy [PFG-

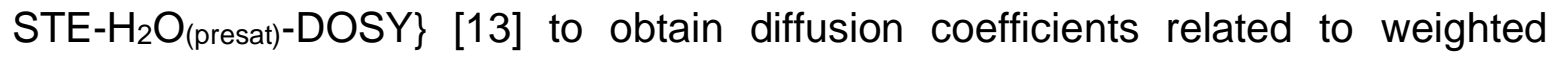
average molecular weights $(\dot{M} w)$ and PDIs of both PAMAM moieties in the solution state. Furthermore, molecular weights (MW) and PDIs obtained with Size-exclusion chromatography (SEC) were orthogonally compared with data obtained with PFGSTE- $\mathrm{H}_{2} \mathrm{O}$ (presat)-DOSY as well as with PDIs from DLS. Also, morphology, distribution pattern, and mechanical characteristics in flat surfaces by atomic force microscopy (AFM) were analyzed. Finally, an analysis of MS-ESI-QToF was employed to obtain the experimental high-energy (10-45 eV) $\mathrm{m} / \mathrm{z}$ fragmentation profiles and compare in a mirror plot the $\mathrm{m} / \mathrm{z}$ shared between OS-PAMAM and PAMAM-G6. 


\section{Materials and methods}

134

135

136

137

138

139

140

141

142

143

144

145

146

147

148

149

150

151

152

153

154

155

156

157

158

159

\subsection{Materials}

The main reactants, methyl acrylate and ethylenediamine (Sigma-Aldrich) were used for the optimized method. The commercial PAMAM dendrimer G6 (PAMAM G6), 5 wt. \% in methanol from Merck, USA was used as control. The methanol used for all the synthesis was purchased in J.T. Baker. For PFG-STE- $\mathrm{H}_{2} \mathrm{O}_{\text {(presat)-DOSY NMR }}$ analysis, deuterium oxide (99.9\% deuteration, CAS No. 7789-20-0) from Merck Millipore was employed. The water used in all the experiments was purified using a Simplicity ${ }^{\circledR}$ UV Water Purification System (Merck Millipore). All chemicals used in this study were analytically pure.

\subsection{Synthesis method}

The synthesis of OS-PAMAM dendrimers is explained in patent application No. MXA2018008247 and was carried by modifying different variables such as the proportion of ethylenediamine and methyl acrylate reactants, stirring speed, and vacuum pressure, which allows the generation of temperature gradients throughout the synthesis. In this way, a polymerization is achieved over time and the Michael addition reactions and amidations are modulated in the same reaction. The reaction was carried out in methanol, under vacuum pressure which generated a decreasing temperature gradient throughout the synthesis from $15^{\circ} \mathrm{C}$ to $5^{\circ} \mathrm{C}$. The vacuum also promotes solvent elimination as well as low molecular weight molecules, that are byproducts of parallel reactions. Another set of impurities was removed by a dialysis membrane of $2000 \mathrm{kDa}$. As a result, a yellow viscous solution was obtained, the reaction yield was $45 \%$, and after the purification step by dialysis, it was $23 \%$ [14].

\subsection{UV-Vis and FT-IR spectroscopy}

The UV-Vis was performed in a spectrophotometer (NanoDrop 2000, Thermo Scientific), $20 \mathrm{mg} / \mathrm{mL}$ of the OS-PAMAM dialyzed and commercial standard PAMAM-G6 were measured in a range from 200 to $800 \mathrm{~nm}$ in type 1 water and 
compared through the two main absorption bands at 210-216 nm and 280-285 nm, corresponding to the backbone structure of secondary and tertiary amides, characteristics of PAMAM structures. Further, both synthesized OS-PAMAM and commercial PAMAM-G6 were dried at $65^{\circ} \mathrm{C}$, and $5 \mathrm{mg}$ of the samples were characterized by Fourier-transform infrared spectra (FT-IR) to obtain the primary and secondary amides, also characteristic functional groups through ATR (Frontier Optica, PerkinEImer, USA).

\subsection{Dynamic Light Scattering}

The particle size distribution, PDI, and ZP were measured by dynamic light scattering (DLS). The three batches of OS-PAMAM dendrimers were filtered by an Acrodisc ${ }^{\circledR}$ Syringe Filter of $0.2 \mu \mathrm{m}$, suspended in methanol (99.9\%) at $20 \mathrm{mg} / \mathrm{mL}$ and stored at 2- $8^{\circ} \mathrm{C}$. All suspensions $(2 \mathrm{~mL})$ were measured at $25^{\circ} \mathrm{C}$, and the average value was obtained after two repetitions with 25 accumulations, taking into count the PDI. Also, the average ZP was obtained after one repetition and five accumulates. These characteristics were monitored through the time (0-4 months) and compared with PAMAM G6 stored at the same temperature, concentration, and analytic method in a Particulate Systems (NanoPlus, GA, USA) equipment.

\subsection{Size Exclusion Chromatography}

The polydispersity of the molecular weight from both OS-PAMAM and PAMAM-G6 dendrimers was determined by size-exclusion chromatography (SEC). A $5 \mu \mathrm{L}-$ volume of both dendrimer samples $(30 \mathrm{mg} / \mathrm{mL})$ was injected into an Acquity UPLC system (Waters $^{\circledR}$; M.A., USA) equipped with an Acquity UPLC BEH SEC $200 \AA$ column $(4.6 \mathrm{~mm} \times 300 \mathrm{~mm}, 1.7 \mu \mathrm{m})\left(\right.$ Waters $\left.^{\circledR}\right)$, which was maintained at $30^{\circ} \mathrm{C}$ during the analysis. The separation was achieved using a $50 \mathrm{mM}$ phosphate/150 mM NaCl buffer solution ( $\mathrm{pH}$ 6.0) as a mobile phase at $0.4 \mathrm{~mL} / \mathrm{min}$ (JT. Baker; N.J., USA). Data were acquired at $215 \mathrm{~nm}$ and processed using the software Empower. The molecular weight was determined by linear regression of the maximum value in absorbance units (AU) based on the MW standard from 1.5 to $670 \mathrm{kDa}$, Cat 1511901 (Biorad, CA, USA). 
203

204

205

206

207

208

209

210

211

212

213

214

215

216

Three batches of OS-PAMAM and G6 were compared using their PDI values. For this purpose, the SEC profile of each sample was exported from Empower ${ }^{\circledR}$ to Excel (Microsoft, WA, USA) and the PDI values were determined using the method described using formulas 1 to 3 [15]:
(1) $\bar{M}_{n}=\frac{\sum_{i}^{n}\left(W_{i}\right) P W_{i}}{\sum_{i}^{n}\left(W_{i} / M_{i}\right) P W_{i}}$
(2) $\bar{M}_{w}=\frac{\sum_{i}^{n}\left(W_{i} M_{i}\right) P W_{i}}{\sum_{i}^{n}\left(W_{i}\right) P W_{i}}$
(3) $P D I=\frac{\overline{\mathrm{M}}_{\mathrm{w}}}{\overline{\mathrm{M}}_{\mathrm{N}}}$

Where $W_{i}$ is the intensity $(\mathrm{AU})$ of the observed size $(\mathrm{kDa}), P W_{i}$ is the percentual intensity (weighted intensity) and $M_{i}$ is the observed size in the SEC analysis, which was determined using a logarithmic equation determined by the retention time of the components of the MW standard. Structural heterogeneity of dendrimer samples was qualitatively computed in terms of a second derivative analysis of the SEC profile (Figure 4B), whereas homogeneous samples will produce a single antiphase chromatogram pattern, whilst a set of several antiphase patterns are expected within second derivative analysis of heterogeneous samples.

\subsection{Atomic Force Microscopy}

The dendrimers OS-PAMAM and PAMAM-G6 were analyzed in a Bioscope Catalyst AFM (Bruker, Germany) to know the shape, size, and mechanical properties (adhesion force and elasticity). In the experimental tests, $30 \mu \mathrm{L}$ of suspension with dendrimers were deposited and dried on a piece of glass slice of dimension $5 \mathrm{~mm} \times$ $5 \mathrm{~mm}$. The glass slide was first cleaned by immersion in ethanol absolute for $20 \mathrm{~min}$, followed by rinsing with distilled water. It was employed a pyramidal tip SNL Bruker of $2 \mathrm{~nm}$ in a $300 \mathrm{~nm}$ ramp size with 0.4 of a trig threshold. Before using the Thermal Tune Method, the deflection sensitivity of the cantilever was determined in a liquid fluid by using the value of the inverse of the slope of the force curve while the cantilever was in contact with a hard glass surface to obtain the force measurements. The averages of the deflection sensitivity (s) and elasticity constant (k) determined at five different points were $16.57 \mathrm{~nm} / \mathrm{V}$ and $0.57 \mathrm{~N} / \mathrm{m}$, respectively, and the peak force setpoint was performed at $7 \mathrm{Nn}$. The images were analyzed by using the NanoScope Analysis 1.5 Software. 
218 


\subsection{NMR - Pulsed Field Gradient Diffusion Ordered Spectroscopy}

For each batch, $540 \mu \mathrm{L}$ of liquid buffered PAMAM-G6 and OS-PAMAM solution were dissolved in 60 uL of $\mathrm{D}_{2} \mathrm{O}$ (99.9\% deuteration, CAS No. 7789-20-0), for having a 9:1 ratio of $\mathrm{H}_{2} \mathrm{O}: \mathrm{D}_{2} \mathrm{O}$ solvent system. All NMR spectra were recorded on a Bruker 600 AVANCE III HD equipped with a $5 \mathrm{~mm} 1 \mathrm{H} / \mathrm{D}$ TXI probe head with a z-gradient. The following set of NMR experiments were conducted at a controlled temperature of $303 \mathrm{~K}$ : a) Standard ${ }^{1} \mathrm{H}$-one dimensional direct-polarization NMR experiments (Figure 8 and F2-projections in Figure 9A) were carried out with 16 transients of 32768 complex points, having recycling delays of $8 \mathrm{~s}$ and with acquisition times of $1700 \mathrm{~ms}$, produced experimental times of 2 minutes 35 seconds per PAMAM dendrimer batch. No apodization function was applied during Fourier Transform for proton spectra depicted in Figures $8 \mathrm{~A}$ and $8 \mathrm{C}$. In contrast a Resolution Enhancement postprocessing was applied for spectra depicted in Figures 8B and 8B with a Lorentz-toGauss line-sharpening apodization; b) acquisition and processing details of PFGSTE- $\mathrm{H}_{2} \mathrm{O}$ (presat)-DOSY have been recently published $[13,16]$, whereas, for the present study, the following key NMR parameters were used: 64 gradient levels with a linear increase from 2 to $98 \%$, a gradient strength up to $54 \mathrm{G} / \mathrm{cm}$ and 8 transients per gradient increase comprising 65536 complex points were used. The diffusion delay $(\Delta)$ was $100 \mathrm{~ms}$, and the length of the square diffusion encoding gradient pulse (ठ) was $4.2 \mathrm{~ms}$ to assure accurate gradient encoding-decoding signal attenuation. No apodization function within the direct F2 dimension was needed for semi-Fourier transformation prior to Inverse-Laplace indirect F1 transformation.

In this present work the comparative analysis between OS-PAMAM and PAMAM G6 was performed by DOSY-NMR to obtain the relative MW. Hence, it was measured the diffusion coefficient (D) of each chemical-shift assigned resonance and related with the hydrodynamic radius by the Stokes-Einstein equation (with stick boundary conditions):

$$
R_{H}=\frac{k_{B} T}{6 \pi \eta D}
$$


247 where $\mathrm{kB}$ is the Boltzmann constant, $\mathrm{T}$ is the temperature (in $\mathrm{K}$ ), and $\eta$ is the viscosity 248 of the solution $\left(\sim 9.7 \mathrm{cP}\right.$ for $\mathrm{D}_{2} \mathrm{O}$ at $\left.30{ }^{\circ} \mathrm{C}\right)[17]$.

249 The obtained diffusion coefficients were internally referenced with respect to the 250 water signal at a value of $-7.629 \times 10^{-10} \mathrm{~m}^{2} \mathrm{~s}^{-1}$ (see figure $8 \mathrm{~A}$, diffusion signal 251 correlating with the water ${ }^{1} \mathrm{H}$ chemical shift at $\left.4.7 \mathrm{ppm}\right)$. Experimental polydispersity 252 indexes in the solution state ( $\boldsymbol{\Xi}$ in figure $8 \mathrm{~A}$ ) in turn related to weight average 253 molecular weights $\left(\dot{\mathbf{M}}_{\mathbf{w}}\right.$ in figure 8A) correlated with the experimental diffusion 254 coefficient distributions at the low concentration regime [13] of both PAMAM-G6 and 255 OS-PAMAM dendrimers are reported as figure legends.

$256 \quad 2.8$ Mass Spectrometry coupled to Liquid Chromatography

257 The PAMAM-G6 and OS-PAMAM samples were prepared at a concentration of 100 258 UM with a 50:50 methanol: acetonitrile mixture. Subsequently, 10 ul of each sample 259 was injected into an Acquity® UPLC Class I system (Waters; MA, USA) equipped 260 with a $1.7 \mu \mathrm{m}(2.1 \mathrm{~mm} \times 100 \mathrm{~mm})$ BEH300 C4 column $45^{\circ} \mathrm{C}$ (Waters®; MA, USA). 261 The samples were eluted using water+formic acid (0.1\%) (phase A) and 262 acetonitrile+formic acid (0.1\%) (phase B) as follows: $100 \%$ of phase A from 0 to 1 $263 \mathrm{~min}$ followed by a linear gradient of $100-0 \%$ from 1 to $11 \mathrm{~min}$ of phase $A$; all reagents 264 used in this analysis were MS grade (Sigma-Aldrich, MO, USA). Then, the mass 265 analysis was performed on a Vionß hybrid Quadrupole Time of Flight Spectrometer equipped with an Electro Spray Ionization (ESI) source. The mass spectrometer was operated in positive polarity and MSE sensitivity mode from 50 to $4000 \mathrm{~m} / \mathrm{z}$; the collision energies were $5.00 \mathrm{eV}$ (low) and $10.00 \mathrm{eV}$ to $45.00 \mathrm{eV}$ (high collision energy ramp) with 2 seconds of scanning time. ESI parameters were set at $140^{\circ} \mathrm{C}$ (source 270 temperature), $600^{\circ} \mathrm{C}$ (desolvation temperature), $30 \mathrm{eV}$ (cone voltage), $100 \mathrm{~L} / \mathrm{h}$ (cone 271 gas), 1,200 L/h (desolvation gas), and 2.75 kV (capillary voltage). A 10-pg/uL [Glu1]272 Fibrinopeptide $B$ solution (Waters $($ ) was infused during the MS analysis at $20 \mu \mathrm{L} / \mathrm{min}$ 273 for mass correction. Data were acquired and processed using UNIFIß software 274 (version 1.9.4.053) (Waters $\AA$ ), on which the chemical formula the PAMAM and their 275 reported fragments [16] were loaded. The $\mathrm{m} / \mathrm{z}$ signals of PAMAM and their fragments 
were searched in the experimental data acquired from the high energy channel considering a 25 ppm error.

\subsection{Statistical analysis}

All the obtained results were determined at least in triplicate and analyzed using the SigmaPlot ${ }^{\circledR}$ software (Systat Software Inc., USA). The averages and Pearson linear regressions were determined using Microsoft Excel ${ }^{\circledR}$, (Microsoft, USA) and differences between groups were analyzed using one-way analysis of variance (ANOVA) and the Student-Newman-Keuls as a post-hoc test. A value of $p \leq 0.05$ was considered statistically significant.

\subsection{UV-Vis and FT-IR spectroscopy of OS-PAMAM and PAMAM-G6}

The OS-PAMAM dendrimers have presented promising applications within the biomedicine field [9]. Therefore, the characteristics and reference values of OSPAMAM dendrimers were determined in comparison to the commercial PAMAM-G6. For that, three OS-PAMAM batches were randomly selected and measured by UVVis. The maximum absorbance at 210-216 $\mathrm{nm}$ and $280-285 \mathrm{~nm}$, corresponding to secondary and tertiary amides, match the wavelengths found in PAMAM-G6 and previous reports, were the height of the peak centered at $\sim 280 \mathrm{~nm}$ changes upon protonation of the intradendrimer tertiary amines $[18,19]$ (Figure 1A). Further, this signal of tertiary amines was consistent through the time up to 16 weeks of observation without significant changes, indicating a complete reaction and stability at $5 \pm 3^{\circ} \mathrm{C}$ (Figure 1B) of a full generation PAMAM dendrimer (Figure 1C).

The functional groups of OS-PAMAM were also compared with PAMAM-G6 by FTIR. The stretching frequencies of OS-PAMAM at $3270 \mathrm{~cm}^{-1}$ of $\mathrm{N}-\mathrm{H}, 1637 \mathrm{~cm}^{-1}$ of $\mathrm{C}=\mathrm{O}, 3089 \mathrm{~cm}^{-1}$ of $\mathrm{C}-\mathrm{H}$, and the $\mathrm{N}-\mathrm{H}$ bend at $1546 \mathrm{~cm}^{-1}$, match with peaks at 3275 $\mathrm{cm}^{-1}, 1637 \mathrm{~cm}^{-1}, 3087 \mathrm{~cm}^{-1}$, and $1547 \mathrm{~cm}^{-1}$ corresponding to secondary amides, 
respectively, of PAMAM-G6 dendrimers (Figure 2). Those peaks have been widely reported for PAMAM dendrimers across different generations $[20,21]$

\subsection{Size, Polydispersity Index and Zeta potential}

To know the OS-PAMAM properties in colloidal solutions and determine its stability at $5 \pm 3{ }^{\circ} \mathrm{C}$, a DLS analysis was performed using the PAMAM-G6 as a reference. The average size was obtained through the hydrodynamic size of three batches of OS-PAMAM and the commercial one; all measurements were performed after the same storage conditions at 0,2 , and 4 months (Table 1 ).

The size was closely related to the PDI, which indicates the heterogeneity of a sample based on size. OS-PAMAM and PAMAM-G6 sized $\sim 10 \mathrm{~nm}$ with PDI of 0.2 - 0.5; approximately $20 \mathrm{~nm}$ when PDI ranges 0.5 - 1.0, and $~ 30 \mathrm{~nm}$ indicates PDI values above the unity (Figure $3 \mathrm{~A}-\mathrm{B}$ ). Therefore, a directly proportional relationship between the size and the PDI for OS-PAMAM and PAMAM-G6 with $\mathrm{R}^{2}=0.9127$ (Figure $3 A$ ) and $R^{2}=0.8278$ (Figure $3 B$ ), respectively, across independent batches and measurements was determined. Although there was no significant increase in the average size or PDI, as a function of storage time in most batches of OS-PAMAM and PAMAM G6, batch 3 presented an increase in polydispersity and size. Polydispersity can occur due to size distribution or agglomeration of the OS-PAMAM during analysis. Other studies have demonstrated that a higher PDI number indicates that NPs are less monodisperse, with a larger size, and will be more likely to form agglomerates over time [22,23]. Furthermore, the ZP of these two NPs was compared, whereas values of $\sim 50 \mathrm{mV}$ were found for PAMAM-G6 and $\sim 14 \mathrm{mV}$ for OS-PAMAM (Figure 3-C and D). The ZP differences between dendrimers could be attributed to the generation since an increase in zeta potential has been observed as a function of generation [24]. However, it should be considered that ZP values are not an absolute measurement of NPs stability. For example, solid lipid nanoparticles with positive ZP have a long-circulating half-life due to the absorption of protein components in the blood, while NPs with negative ZP can be cleared by the reticuloendothelial system. Furthermore, this parameter is very important for biomedical applications because toxicity has been linearly correlated with high 
positive values of ZP $[25,26]$. Indeed, controlling and validating these parameters from the synthesis is crucial for the useful applications of dendrimeric NPs in different fields $[27,28]$.

\subsection{Molecular weight comparisons of OS-PAMAM and PAMAM-G6}

338 The molecular weight (MW), and reproducibility between batches were measured by 339 size exclusion chromatography (SEC). All batches of OS-PAMAM (green, blue and 340 orange chromatograms, figure 4) and PAMAM-G6 (black, Figure 4) respectively 341 present a maximum in their chromatograms at $4.55 \mathrm{~min}$ and 3.2 minutes. Both MW 342 were calculated by linear regression of the MW marker curve (red in figure 4A), 343 resulting in $8.5 \mathrm{kDa}$ and $86 \mathrm{kDa}$ for the three OS-PAMAM batches and PAMAM-G6 344 correspondingly (Figure 4A). The MW of PAMAM-G6 was higher in comparison to 345 the theoretical MW reported (58,048 kDa). Differences between theoretical and 346 experimental MW values of the dendrimers may be associated with various 347 phenomena, such as nonspecific interactions between PAMAM-G6 and SEC column 348 [29]. According to other reports, the intrinsic viscosity of the dendrimers decreases 349 as generation increases, which could affect the measurement of low and high 350 generations with the same analytical method, i.e., G3 vs G6 [30,31].

351 The average PDI obtained in the SEC analysis for OS-PAMAM batches was $1.05 \pm$ 3520.039 , whilst a polydispersity index of 3.16 was computed for PAMAM-G6 (Table 2), in 353 clear agreement with the overestimated $86 \mathrm{kDa}$ SEC molecular weight of said 354 dendrimer. Interestingly, the SEC distribution pattern is wider in OS-PAMAM, 355 suggesting a larger structural heterogeneity. In contrast, the chromatographic peak of the PAMAM-G6 -with a sharper chromatogram linewidth- indicates structural 357 homogeneity of the more dispersed dendrimer. This physicochemical difference 358 amongst products is unambiguously revealed when their SEC profiles are subjected 359 to a Second Derivative Analysis ( $\Delta \Delta$ Absorbance, Figure $4 \mathrm{~B})$ : A single antiphase 360 signal is revealed for the structurally homogeneous PAMAM-G6 dendrimer, as a 361 result of deconvoluting the SEC chromatogram with the $\Delta \Delta$ Absorbance procedure 362 For the contrary, the $\Delta \Delta$ Absorbance deconvolution of broad OS-PAMAM 363 chromatographic SEC peaks produces a more complex pattern, as the result of a 
weighted sum of several antiphase peaks, as a consequence of the structural heterogeneity that One-Step PAMAM moieties are presenting. To the best of our knowledge, this post-processing SEC treatment has not been presented so far as a qualitative method for revealing structural heterogeneity of polyamidoamide dendrimers. In addition, when OS-PAMAM and PAMAM-G6 are analyzed by SEC at $\mathrm{pH} 2.5$ both products reduce their hydrodynamic ratio as both polydispersions elute at shorter retention times [15] but the peak of OS-PAMAM widens whereas PAMAMG6 gets sharper. This suggests that OS-PAMAM polydispersity includes several structures with different pKa whereas PAMAM-G6 has a reduced number of structures with a closer pKa among them. Besides, OS-PAMAM may include structures different to PAMAM. A previous MW report of commercial PAMAM G5 by SEC has indicated the presence of trailing generation impurities and oligomerized defects, like for instance, non-ideal amidation, that can represent approximately $30 \%$ of the sample by weight (Figure S1) [32]. For instance, in comparisons, the higher peak should be taken as the full-body PAMAM structure [33,34].

Despite the structural heterogeneity of OS-PAMAM, the three tested batches evinced the same behavior, which may facilitate the reproducibility of its biologic application (Figure S2) [35,36]. Several studies have demonstrated the encapsulation efficiency as well as biocompatibility of highly dendronized polymers, indicating its potential biomedical application [37-39].

\subsection{Comparative analysis of size, shape, and force measurements by AFM}

The shape of OS-PAMAM and PAMAM-G6 was compared by AFM. The images showed the distribution over the slide for both NPS. The average size was measured

387 through a maximum vertical radius $(\mathrm{Rz})$ of cross-sections in particles analyzed in all 388 images from different fields over the slide. A similar Rz (height) of $14.21 \pm 2.81 \mathrm{~nm}$ 389 and $13.30 \pm 2.56 \mathrm{~nm}$ for OS-PAMAM and PAMAM-G6, respectively, were obtained 390 (Figure 5A-B). Further, a mostly spherical shape for both NPs was observed, as 391 reported in other studies [40,41]. However, superficial irregularities in OS-PAMAM 392 dendrimers were observed, suggesting the presence of cavities formed between 
ramifications which explain the minor MW of OS-PAMAM, and the collapsed structure on the slide, which can form agglomerates (Figure 4, 5-C).

The force measurements of OS-PAMAM and PAMAM G6 dendrimer was also obtained by AFM, comparing the differences in adhesion and elasticity (DMT Modulus) between the surface of the glass and each nanomaterial. The analysis was performed by pushing the AFM tip onto the surface of the samples and monitoring force-versus-distance curves. Automatic analysis of curves using NanoScope Analysis Software generates maps of mechanical properties distribution with topographical imaging [42] (Figure 6A-7A). The highest value of several crosssections of adhesion $(\mathrm{nN})$ and elasticity $(\mathrm{MPa})$ from $50 \mathrm{NPs}$ was average. For adhesion, the average Rz of OS-PAMAM and PAMAM-G6 were $21.878 \pm 3.67 \mathrm{nN}$ and $18.18 \pm 3.55 \mathrm{nN}$, respectively, and they did not present significant differences and the interaction between the dendrimers and the AFM tip employed were similar (Figure 6B). The OS-PAMAM presented a larger adhesion force than reported for PAMAM-G4-NH 2 with different tip substrate [43]. Adhesion between the polymer surface and AFM-tip is controlled by the chemical groups at or near the surface. Despite the low MW of OS-PAMAM on comparison to PAMAM G6, the similar adhesion force could be due to related surface functional groups between both dendrimers $[44,45]$. Dendrimers in biomedical applications interact with cargo molecules and cells, and the force of adhesion provides the fundamental basis for the hypothetical notion that high binding avidity [46].

Regarding the elasticity in Derjaguin-Muller-Toporov (DMT) modulus maps, the samples themselves are softer and thickness than the glass. Differential $R z$ average values were $27.69 \pm 3.11 \mathrm{MPa}$ and $37.80 \pm 4.42 \mathrm{MPa}$ for OS-PAMAM and PAMAMG6, respectively, with significant differences between dendrimers (Figure 7A). In general, the OS-PAMAM DMT modulus values decreased significantly compared with PAMAM-G6 (Figure 7B). An increase in elasticity concerning the molecular weight and density of the NPs has been observed [42,47]. Besides, a much higher elastic modulus has been observed for individual G4 than G3 dendrimers molecules due to their more shape persistent conformation [48]. Hence some biophysical 
characteristics of OS-PAMAM aggregates surfaces given by the adhesion were comparable to PAMAM-G6. Otherwise, the DMT modulus differences suggest that OS-PAMAM has a more flexible conformation than PAMAM-G6.

\subsection{Structural analysis, diffusion coefficient distributions, weighted average} molecular weight, polydispersity indexes and structural homogeneity of OSPAMAM and PAMAM-G6 by Pulsed-Field Gradient Diffusion Ordered (PFGDOSY) NMR spectroscopy

OS-PAMAM dendrimers were synthesized with the use of a modified Michael addition method between ethylenediamine and acrylic acid methyl ester; commonly, PAMAM has polar pockets comprised mainly of alkyl chains, tertiary amines, and amido scaffolds [14]. Proton nuclear magnetic resonance ( ${ }^{1} \mathrm{H}$ NMR) has provided a robust analysis for structural studies and identity evaluation of PAMAM dendrimers. Also, PAMAM-drug hosts interactions were previously analyzed by ${ }^{1} \mathrm{H}$ NMR titrations [49] through dipolar NOESY correlation-exchanges [50] and PFG NMR [51] ${ }^{1} \mathrm{H}$ NMR Chemical shift assignment of PAMAM dendrimer has been described [52]: $2.39 \mathrm{ppm}$ (120H, br, $\left.-\mathrm{NCH}_{2} \mathrm{CH}_{2} \mathrm{CONH}-, \mathrm{Ha}\right) ; 2.60$ ppm (56H, br, $-\mathrm{CONH} \mathrm{CH}_{2} \mathrm{CH}_{2} \mathrm{~N}-, \mathrm{Hb}$ ); 2.69 ppm (120H, br, $\left.-\mathrm{NCH}_{2} \mathrm{CH}_{2} \mathrm{CONH}-, \mathrm{Hc}\right) ; 2.79 \mathrm{ppm}\left(64 \mathrm{H}, \mathrm{br},-\mathrm{CONHCH}_{2} \mathrm{CH}_{2}\right.$ $\left.\mathrm{NH}_{2}, \mathrm{Hb}^{\prime}\right) ; 3.21 \mathrm{ppm}\left(56 \mathrm{H}, \mathrm{br},-\mathrm{CONHCH}_{2} \mathrm{CH}_{2} \mathrm{~N}-, \mathrm{Hd}\right) ; 3.26 \mathrm{ppm}(64 \mathrm{H}$, br, $-\mathrm{CONHCH}_{2} \mathrm{CH}_{2} \mathrm{NH}_{2}, \mathrm{Hd}^{\prime}$ ).

First, standard ${ }^{1} \mathrm{H}$-one dimensional direct-polarization NMR spectra of commercial PAMAM-G6 (Figure 8A and B) and OS-PAMAM (Figure 8C and D) with identical post-processing treatment, reveal subtilities comprising structural heterogeneity and polydispersity of both samples. Spectra from figures $8 \mathrm{~A}$ and $8 \mathrm{C}$ were only Fourier Transformed with no post-processing treatment, whilst Figures $8 \mathrm{~B}$ and $8 \mathrm{D}$ were apodized with a Lorentz-to-Gauss line sharpening of $6 \mathrm{~Hz}$ and a Gaussian center position at $20 \%$ of the Free Induction Decay (FID) for a resolution enhancement, despite a dramatic loose of sensitivity. In a first glance, ${ }^{1} \mathrm{H}$-one dimensional directpolarization NMR spectra of PAMAM-G6 with no application of any weighting 
function (Figure 8A), reveal linewidths at half height (LWHH) of $15.71 \mathrm{~Hz}$ for $-\mathrm{CONH}$ $\mathrm{CH}_{2} \mathrm{CH}_{2} \mathrm{~N}$ - resonances at $\delta=3.39 \mathrm{ppm}$ and $3.025 \mathrm{ppm}$, and even broader $\mathrm{LWHHs}$ of $63.72 \mathrm{~Hz}$ ( $\delta=2.78 \mathrm{ppm},-\mathrm{CONHCH} \mathrm{CH}_{2} \mathrm{~N}$ - spin system), $56.74 \mathrm{~Hz}$ ( $\delta=2.59 \mathrm{ppm},-\mathrm{NCH}_{2}$ $\mathrm{CH}_{2} \mathrm{CONH}-$ spin system), $20.95 \mathrm{~Hz}\left(\delta=2.42 \mathrm{ppm},-\mathrm{CONH} \mathrm{CH}_{2} \mathrm{CH}_{2} \mathrm{~N}-\right.$ spin system) and $21.82 \mathrm{~Hz}$ ( $\delta=2.34 \mathrm{ppm},-\mathrm{NCH}_{2} \mathrm{CH}_{2} \mathrm{CONH}-$ spin system). Broader ${ }^{1} \mathrm{H}$ LWHH in OSPAMAM spectrum with no post-processing (Figure $8 \mathrm{C}$ ) does not have an origin from higher polydispersity but rather due to higher structural heterogeneity [35] as unambiguously revealed in Figure 8D, in comparison to Figure 8B. Resolution enhancement achieved with identical Lorentz-to-Gauss weighting functions in both dendrimers ${ }^{1} \mathrm{H}$ NMR spectra produces different results. Line sharpening apodization applied to PAMAM-G6 proton spectrum produces equivalent LWWHs (Figure 8B) as when no apodization function was applied (Figure 8A). In contrast, broad resonances of OS-PAMAM ${ }^{1} \mathrm{H}$ NMR spectrum (Figure $8 \mathrm{C}$ ) are deconvoluted into narrower frequencies of c.a. 1 to $1.5 \mathrm{~Hz}$ of LWHHs (Figure 8D) when resolution enhancement is achieved with a Lorentz-to-Gauss apodization function identically applied as in PAMAM-G6 ${ }^{1} \mathrm{H}$ spectrum (Figure 8B). The set of narrow resonances revealed in Figure 8D strongly suggest that OS-PAMAM presents an important structural heterogeneity due to defects in the dendrimer structure during the synthesis (Section 2.2) and a lack of dendrimer purity such as with the commercial PAMAM-G6.

Analysis of two-dimensional PFG-STE-H2O(presat)-DOSY spectra of both dendrimers demonstrate at first glance, differences in diffusion coefficient distributions between both dendrimers: the OS-PAMAM showed higher diffusion coefficient distributions around a lower logarithmic diffusion coefficient average of Log DC av $=-8.8 \mathrm{~m}^{2} \mathrm{~s}^{-1} \pm 0.05$ than the PAMAM-G6 counterpart $\left(\log \mathbf{D C}_{\mathrm{av}}=-9.6 \pm\right.$ $0.05 \mathrm{~m}^{2} \mathrm{~s}^{-1}$, Figure 9A). Furthermore, experimental solution-state DOSY-MW distributions obtained from diffusion coefficient distributions [13] are represented as histograms (Figure 9B) and expressed in Da for PAMAM dendrimers ranging from G1 to G6. PAMAM-G6 weighted average molecular weight of $M W=55711.86 \pm 0.7$ corresponds to the theoretical value elsewhere reported [53], whilst OS-PAMAM weighted average molecular weight of $\mathrm{MW}=5056.47 \pm 0.7$ strongly suggest the 
484

485

486

487

488

489

490

491

492

493

494

495

496

497

498

499

500

501

502

503

504

505

506

507

508

509

510

511

512

513

514

presence of a PAMAM-G3 dendrimer, in full agreement with SEC data, predicting an average MW of $8.5 \mathrm{kDa}$ for OS-PAMAM (Figure 4). Finally, solution-state polydispersity indexes (PDI, $\oplus$ in figure $9 \mathrm{~A}$ ) are experimentally obtained from the molecular weight distributions at the low concentration regime from the experimental average diffusion coefficient distributions ( $\log \mathbf{D C}_{\mathbf{a v}}$ in Figure 9A) and the width of each distribution, represented by the experimental diffusion coefficient distributions [13]. Table 2 resumes the solution-state MW and PDIs of OS-PAMAM and PAMAMG6 obtained with non-invasive DOSY-NMR spectroscopy for DLS (Table 1) and SEC (Figure 4), finding orthogonality amongst the three techniques. Present results are in agreement with other studies reporting that PAMAM G3 is around $7 \mathrm{kDa}[29,54]$. Moreover, lower logarithmic diffusion coefficient average of OS-PAMAM (Log DC $a v$ $\left.=-8.8 \mathrm{~m}^{2} \mathrm{~s}^{-1} \pm 0.05\right)$ that in turn weights higher diffusion coefficient distributions responsible to produce lower PDI and average MW values, with respect PAMAMG6, as revealed with DLS, SEC and PFG DOSY NMR (Table 2), in combination with the observed set of narrow resonances in ${ }^{1} \mathrm{H}$ NMR spectrum apodized with a line sharpening weighting function (Figure 8D), allows to conclude that herein produced OS-PAMAM presents lower polydispersity but higher structural heterogeneity, with respect its more polydisperse but more homogeneous PAMAM-G6 counterpart.

In complement, the molecular weight of G4 dendrimer is $14195 \mathrm{Da}$ measured by MALDI-TOF mass spectrometry, whereas the associated polydispersity index (PDI) of the said dendrimer is 1.01 , obtained by gel permeation chromatography [51]. All these data suggest that the molecular weight of OS-PAMAM dendrimer equates to a PAMAM-G3.

\subsection{Structural characterization by MS-MS}

As part of the physicochemical characterization of OS-PAMAM and PAMAM-G6, and taking advantage of the sensitivity of MS-ESI-QToF, we obtained their experimental high-energy (10-45 eV) $\mathrm{m} / \mathrm{z}$ fragmentation profiles and compared in a mirror plot, as shown in Figure 10. It was found that in the range of $50-600 \mathrm{~m} / \mathrm{z}$ both samples share, among other masses, the values of $\mathrm{m} / \mathrm{z} 571.4013,457.3241,367.2433$, 
355.2460, 343.2431, 325.2336, 241.1646, 229.1643, 199.1542, 127.0851, 98.0609 and 85.0761 . These $\mathrm{m} / \mathrm{z}$ values belong to the decomposition pathway by retroMichael reactions for the species $[\mathrm{M}+5 \mathrm{H}] 5+,[\mathrm{M}+7 \mathrm{H}] 7+$ and $[\mathrm{M}+9 \mathrm{H}] 9+$ of the PAMAMs of G1 G2 and G3 respectively. This correspondence with the values calculated for the ionic precursors and the path of the fragments for G0 (Table of Figure 10) demonstrate the identity of OS-PAMAM. On the other hand, in the enlarged section of Figure 10, from 471 to $482 \mathrm{~m} / \mathrm{z}$, the species of $479.0647 \mathrm{~m} / \mathrm{z}$ reported for the intramolecular cyclization of the ester group was not found, which is one of the typical side-products of the Michael addition. This indicates that during the synthesis of OS-PAMAM cyclic side-product are not formed [55].

In addition, to determine the reproducibility between OS-PAMAM batches the highenergy $\mathrm{m} / \mathrm{z}$ profiles of batch 1 and 2 were compared. It was found that both batches are highly similar containing charge mass values of the characteristic fragments of the G0, G1, G2 and G3 dendrimers at the same relative intensity (Figure S3). The identification of the most abundant fragments of OS-PAMAM will be useful for biomedical applications such as for determining the elimination pathways in pharmacokinetic surveys using urine as the main elimination via $([26,56,57]$.

\section{Conclusion}

In summary, we performed a detailed physicochemical characterization of OSPAMAM compared with commercial PAMAM-G6. Our finding reveals that OSPAMAM is a dendrimer with lower polydispersity but higher structural heterogeneity, with respect its G6 commercial counterpart. Size exclusion chromatography and nuclear magnetic resonance spectroscopy have found excellent orthogonality to demonstrate the polydispersity and structural homogeneity of herein analyzed dendrimers. For the first time, two postprocessing treatments $-\Delta \Delta$ Absorbance in SEC and Lorentz-to-Gauss resolution enhancements in NMR spectra have revealed by their principles, the structural heterogeneity of herein produced OS-PAMAM, that could in turn be used to reveal defects within the dendrimer structure as well as the 
purity of novel or traditional PAMAM synthetic approaches. Interestingly, OSPAMAM structural heterogeneity and polydispersity is conserved amongst batches. That relation not only remains between repetitions but also over time up to 4 months at $5 \pm 3{ }^{\circ} \mathrm{C}$. In general, the ZP of OS-PAMAM were less positive than PAMAM-G6. However, ZP was consistent between batches and time for both dendrimers. The AFM morphological analysis showed a circular shape in all dendrimers, and cavities were detected in OS-PAMAM. Further, the adhesion force did not present significant differences, while the elasticity (DMT Modulus) was higher in PAMAM-G6, which represents a similar chemical surface but a different internal structure. The MW of OS-PAMAM is around 10 times smaller than commercial G6, as orthogonally detected by SEC and PFG-STE-H2O(presat)-DOSY, whereas lower OS-PAMAM masses are explained due to their cavities. OS-PAMAM / PAMAM-G6 MS-MS mirror plots demonstrate chemical equivalency amongst herein analyzed dendrimers, with a slight advantage that OS-PAMAM is produced with a faster and low-cost synthetic protocol. These novel NPs has been previously studied as a drug delivery system [11]; however, more biological studies will be carried out to compare the biomedical applicability with commercial PAMAM, taking into account the structural heterogeneity, polydispersity and synthetic reproducibility.

\section{Glossary}

OS-PAMAM: PAMAM dendrimers obtained by a One-Step method of synthesis

\section{Acknowledgments}

This project was financed by Secretaría de Investigación y Posgrado (SIP-IPN) through projects INSIGNIA, 20202051, 20210781 and Student Innovation project of Instituto Politécnico Nacional: 201952. The authors were grateful for the laboratory and the equipment support from Laboratorio Nacional de Ciencia, Tecnología y Gestión Integrada del Agua (LNAGUA) at CICATA-IPN. S.A.T.P. were awarded with CONACyT. E.R.G. is COFAA, EDI, and SNI grant fellow. L.G.Z.V thanks CONACYTMéxico for grant INFRA-2016 N²69012. J.E.H-P. acknowledge the Mexican Ministry of Science and Technology (Consejo Nacional de Ciencia y Tecnología, 
573 CONACyT) junior research funding program No. 682 "Cátedras, CONACyT, 574 Laboratorio Nacional de Investigación y Servicio Agroalimentario y Forestal”. The 575 SEC analysis were performed using the equipment and facilities of "Laboratorio

576 Nacional para Servicios Especializados de Investigación, Desarrollo e Innovación (I 577 + D + i) para Farmoquímicos y Biotecnológicos" (LANSEIDI-FarBiotec-CONACyT), 578 which is part of "Unidad de Desarrollo e Investigacioín en Bioprocesos (UDIBI)-IPN". 579 580

The author reports no conflicts of interest in this work.

\section{Disclosure}

583

584

585

586

587

588

589

590

591

592

593

594

595

596

597

598

599

600

601

\section{References}

[1] D. Kaur, K. Jain, N.K. Mehra, P. Kesharwani, N.K. Jain, A review on comparative study of PPI and PAMAM dendrimers, J. Nanoparticle Res. 18 (2016) 146. https://doi.org/10.1007/s11051-016-3423-0.

[2] S. Duinkerken, S.K. Horrevorts, H. Kalay, M. Ambrosini, L. Rutte, T.D. de Gruijl, J.J. Garcia-Vallejo, Y. van Kooyk, Glyco-Dendrimers as Intradermal Anti-Tumor Vaccine Targeting Multiple Skin DC Subsets, Theranostics. 9 (2019) 5797-5809. https://doi.org/10.7150/thno.35059.

[3] S. Svenson, D.A. Tomalia, Dendrimers in biomedical applications-reflections on the field, Adv. Drug Deliv. Rev. $64 \quad$ (2012) 102-115. https://doi.org/10.1016/j.addr.2012.09.030.

[4] E. Abbasi, S.F. Aval, A. Akbarzadeh, M. Milani, H.T. Nasrabadi, S.W. Joo, Y. Hanifehpour, K. Nejati-Koshki, R. Pashaei-Asl, Dendrimers: synthesis, applications, and properties, Nanoscale Res. Lett. 9 (2014) 247. https://doi.org/10.1186/1556-276X-9247.

[5] Z. Lyu, L. Ding, A.Y.-T. Huang, C.-L. Kao, L. Peng, Poly(amidoamine) dendrimers: covalent and supramolecular synthesis, Mater. Today Chem. 13 (2019) 34-48. https://doi.org/10.1016/j.mtchem.2019.04.004.

[6] A.S. Ertürk, M.U. Gürbüz, M. Tülü, A.E. Bozdoğan, Water-soluble TRIS-terminated PAMAM dendrimers: microwave-assisted synthesis, characterization and $\mathrm{Cu}(\mathrm{II})$ 
intradendrimer complexes, RSC Adv. 5 (2015) 60581-60595. https://doi.org/10.1039/C5RA11157A.

604 [7] J. López-Andarias, J. Guerra, G. Castañeda, S. Merino, V. Ceña, P. Sánchez-Verdú, 605 Development of Microwave-Assisted Reactions for PAMAM Dendrimer Synthesis, Eur. J. Org. Chem. 2012 (2012) 2331-2337. https://doi.org/10.1002/ejoc.201101717.

[8] M.V. Walter, M. Malkoch, Simplifying the synthesis of dendrimers: accelerated approaches, Chem. Soc. Rev. $41 \quad$ (2012) 4593-4609. https://doi.org/10.1039/C2CS35062A.

[9] S.M. Grayson, J.M.J. Fréchet, Convergent Dendrons and Dendrimers: from Synthesis to Applications, Chem. Rev. 101 (2001) 3819-3868. https://doi.org/10.1021/cr990116h. [10]M. Arseneault, C. Wafer, J.-F. Morin, Recent Advances in Click Chemistry Applied to Dendrimer Synthesis, $\quad$ Molecules. $20 \quad$ (2015) 9263-9294. https://doi.org/10.3390/molecules20059263.

[11]S.A. Torres-Pérez, M. del P. Ramos-Godínez, E. Ramón-Gallegos, Glycosylated onestep PAMAM dendrimers loaded with methotrexate for target therapy in breast cancer cells MDA-MB-231, J. Drug Deliv. Sci. Technol. 58 (2020) 101769. https://doi.org/10.1016/j.jddst.2020.101769.

[12]J. Li, H. Liang, J. Liu, Z. Wang, Poly (amidoamine) (PAMAM) dendrimer mediated delivery of drug and pDNA/siRNA for cancer therapy, Int. J. Pharm. 546 (2018) 215225. https://doi.org/10.1016/j.jpharm.2018.05.045.

[13]J.E. Herbert-Pucheta, C.A. López-Morales, E. Medina-Rivero, S. Estrada-Parra, S.M. Pérez-Tapia, L.G. Zepeda-Vallejo, Consistency of a dialyzable leucocyte extract manufactured at GMP facilities by nuclear magnetic resonance spectroscopy, J. Pharm. Biomed. Anal. 196 (2021) 113940. https://doi.org/10.1016/j.jpba.2021.113940.

[14] espacenetDocument.pdf, (n.d.). https://worldwide.espacenet.com/data/espacenetDocument.pdf?flavour=plainPage\&loc ale $=$ en_EP \&FT $=D \&$ date $=20200106 \& C C=M X \& N R=2018008247 A \& K C=A \&$ pageNumbe $\mathrm{r}=1$ \&\#view=Fit\&toolbar=0\&statusbar=0\&messages=0\&navpanes=0\&scrollbar=0 (accessed October 5, 2021).

[15]A. Gupta, S. Dubey, M. Mishra, Unique Structures, Properties and Applications of Dendrimers, J. Drug Deliv. Ther. 8 (2018) 328-339. https://doi.org/10.22270/jddt.v8i6s.2083.

[16]S. Vázquez-Leyva, L. Vallejo-Castillo, C.A. López-Morales, J.E. Herbert-Pucheta, L.G. Zepeda-Vallejo, M. Velasco-Velázquez, L. Pavón, S.M. Pérez-Tapia, E. Medina-Rivero, 
636

637

638

639

640

641

642

643

644

645

646

647

648

649

650

651

652

653

654

655

656

657

658

659

660

661

662

663

664

665

666

667

668

669

Identity Profiling of Complex Mixtures of Peptide Products by Structural and Mass Mobility Orthogonal Analysis, Anal. Chem. 91 (2019) 14392-14400. https://doi.org/10.1021/acs.analchem.9b02873.

[17]R.C. Hardy, R.L. Cottington, Viscosity of deuterium oxide and water in the range 5 to 125 C, J. Res. Natl. Bur. Stand. 42 (1949) 573. https://doi.org/10.6028/jres.042.049.

[18]S. Pande, R.M. Crooks, Analysis of Poly(amidoamine) Dendrimer Structure by UV-Vis Spectroscopy, Langmuir. 27 (2011) 9609-9613. https://doi.org/10.1021/la201882t.

[19]K.J. Shah, T. Imae, A. Shukla, Selective capture of $\mathrm{CO}_{2}$ by poly(amido amine) dendrimer-loaded organoclays, RSC Adv. 5 (2015) 35985-35992. https://doi.org/10.1039/C5RA04904K.

[20]Y. Zhang, X. Liu, L. Li, Z. Guo, Z. Xue, X. Lu, An electrochemical paracetamol sensor based on layer-by-layer covalent attachment of MWCNTs and a G4.0 PAMAM modified GCE, Anal. Methods. 8 (2016) 2218-2225. https://doi.org/10.1039/C5AY03241E.

[21]E. Vasile, A. Serafim, D. Petre, D. Giol, P. Dubruel, H. lovu, I.C. Stancu, Direct Synthesis and Morphological Characterization of Gold-Dendrimer Nanocomposites Prepared Using PAMAM Succinamic Acid Dendrimers: Preliminary Study of the Calcification Potential, Sci. World J. 2014 (2014) 1-15. https://doi.org/10.1155/2014/103462.

[22]J. Viéville, M. Tanty, M.-A. Delsuc, Polydispersity index of polymers revealed by DOSY NMR, J. Magn. Reson. 212 (2011) 169-173. https://doi.org/10.1016/j.jmr.2011.06.020.

[23]T. Mudalige, H. Qu, D. Van Haute, S.M. Ansar, A. Paredes, T. Ingle, Chapter 11 Characterization of Nanomaterials: Tools and Challenges, in: A. López Rubio, M.J. Fabra Rovira, M. martínez Sanz, L.G. Gómez-Mascaraque (Eds.), Nanomater. Food Appl., Elsevier, 2019: pp. 313-353. https://doi.org/10.1016/B978-0-12-814130-4.000117.

[24]C.S. Braun, J.A. Vetro, D.A. Tomalia, G.S. Koe, J.G. Koe, C. Russell Middaugh, Structure/Function Relationships of Polyamidoamine/DNA Dendrimers as Gene Delivery Vehicles, J. Pharm. Sci. 94 (2005) 423-436. https://doi.org/10.1002/jps.20251. [25]S.P. Mukherjee, M. Davoren, H.J. Byrne, In vitro mammalian cytotoxicological study of PAMAM dendrimers - Towards quantitative structure activity relationships, Toxicol. In Vitro. 24 (2010) 169-177. https://doi.org/10.1016/j.tiv.2009.09.014.

[26]D.H. Nguyen, L.G. Bach, D.-H. Nguyen Tran, V.D. Cao, T.N.Q. Nguyen, T.T.H. Le, T.T. Tran, T.T.H. Thi, Partial Surface Modification of Low Generation Polyamidoamine Dendrimers: Gaining Insight into their Potential for Improved Carboplatin Delivery, Biomolecules. 9 (2019) 214. https://doi.org/10.3390/biom9060214. 
670

671

672

673

674

675

676

677

678

679

680

681

682

683

684

685

686

687

688

689

690

691

692

693

694

695

696

697

698

699

700

701

702

[27]S. Chandra, M. Mayer, A.J. Baeumner, PAMAM dendrimers: A multifunctional nanomaterial for ECL biosensors, Talanta. 168 (2017) 126-129. https://doi.org/10.1016/j.talanta.2017.03.016.

[28]M. Danaei, M. Dehghankhold, S. Ataei, F. Hasanzadeh Davarani, R. Javanmard, A. Dokhani, S. Khorasani, M. Mozafari, Impact of Particle Size and Polydispersity Index on the Clinical Applications of Lipidic Nanocarrier Systems, Pharmaceutics. 10 (2018) 57. https://doi.org/10.3390/pharmaceutics10020057.

[29]M.A. van Dongen, A. Desai, B.G. Orr, J.R. Baker, M.M. Banaszak Holl, Quantitative analysis of generation and branch defects in G5 poly(amidoamine) dendrimer, Polymer. 54 (2013) 4126-4133. https://doi.org/10.1016/j.polymer.2013.05.062.

[30]M. Czok, A.M. Katti, G. Guiochon, Effect of sample viscosity in high-performance sizeexclusion chromatography and its control, J. Chromatogr. A. 550 (1991) 705-719. https://doi.org/10.1016/S0021-9673(01)88576-9.

[31]A.M. Striegel, Viscometric Detection in Size-Exclusion Chromatography: Principles and Select Applications, Chromatographia. $79 \quad$ (2016) 945-960. https://doi.org/10.1007/s10337-016-3078-0.

[32]M.A. van Dongen, B.G. Orr, M.M. Banaszak Holl, Diffusion NMR Study of GenerationFive PAMAM Dendrimer Materials, J. Phys. Chem. B. 118 (2014) 7195-7202. https://doi.org/10.1021/jp504059p.

[33]W.G. Lesniak, M.S.T. Kariapper, B.M. Nair, W. Tan, A. Hutson, L.P. Balogh, M.K. Khan, Synthesis and Characterization of PAMAM Dendrimer-Based Multifunctional Nanodevices for Targeting $\alpha_{\vee} \beta_{3}$ Integrins, Bioconjug. Chem. 18 (2007) 1148-1154. https://doi.org/10.1021/bc070008z.

[34]M. Ficker, V. Paolucci, J.B. Christensen, Improved large-scale synthesis and characterization of small and medium generation PAMAM dendrimers, Can. J. Chem. 95 (2017) 954-964. https://doi.org/10.1139/cjc-2017-0108.

[35]V. Torres-Salas, B.E. Hernández-Rodríguez, A. Hernández-Montes, E. Castillo, H. Zuleta-Prada, J.E. Herbert-Pucheta, Solid-state NMR spectroscopy for disentangling structural and motional features of lyophilized ripened cheese water-soluble extracts related to antimicrobial activity, Food Chem. 334 (2021) 127603. https://doi.org/10.1016/j.foodchem.2020.127603.

[36]C.C. Lee, J.A. MacKay, J.M.J. Fréchet, F.C. Szoka, Designing dendrimers for biological applications, Nat. Biotechnol. 23 (2005) 1517-1526. https://doi.org/10.1038/nbt1171. 
703

704

705

706

707

708

709

710

711

712

713

714

715

716

717

718

719

720

721

722

723

724

725

726

727

728

729

730

731

732

733

734

735

[37]M. Kumari, S. Gupta, K. Achazi, C. Böttcher, J. Khandare, S.K. Sharma, R. Haag, Dendronized Multifunctional Amphiphilic Polymers as Efficient Nanocarriers for Biomedical Applications, Macromol. Rapid Commun. 36 (2015) 254-261. https://doi.org/10.1002/marc.201400467.

[38]A. Kesavan, P. Ilaiyaraja, W. Sofi Beaula, V. Veena Kumari, J. Sugin Lal, C. Arunkumar, G. Anjana, S. Srinivas, A. Ramesh, S.K. Rayala, D. Ponraju, G. Venkatraman, Tumor targeting using polyamidoamine dendrimer-cisplatin nanoparticles functionalized with diglycolamic acid and herceptin, Eur. J. Pharm. Biopharm. 96 (2015) 255-263. https://doi.org/10.1016/j.ejpb.2015.08.001.

[39]A.A. Chis, C. Dobrea, C. Morgovan, A.M. Arseniu, L.L. Rus, A. Butuca, A.M. Juncan, M. Totan, A.L. Vonica-Tincu, G. Cormos, A.C. Muntean, M.L. Muresan, F.G. Gligor, A. Frum, Applications and Limitations of Dendrimers in Biomedicine, Molecules. 25 (2020) 3982. https://doi.org/10.3390/molecules25173982.

[40]O. Valdés, C. Vergara, F.M. Nachtigall, Z. Lopez-Cabaña, J. Tapia, L.S. Santos, Pamam built-on-silicon wafer thin-layer extraction devices for selective metal contamination detection, Tetrahedron Lett. $57 \quad$ (2016) 2468-2473. https://doi.org/10.1016/j.tetlet.2016.04.063.

[41]A. Barzegar Behrooz, F. Nabavizadeh, J. Adiban, M. Shafiee Ardestani, R. Vahabpour, M.R. Aghasadeghi, H. Sohanaki, Smart bomb AS1411 aptamer-functionalized/PAMAM dendrimer nanocarriers for targeted drug delivery in the treatment of gastric cancer, Clin. Exp. Pharmacol. Physiol. 44 (2017) 41-51. https://doi.org/10.1111/1440-1681.12670.

[42]M. Hussain, A. Memic, N. Alothmany, R. Al-Dhaheri, F. Al-hazmi, H. Alhadrami, A. Khademhosseini, Characterization of Fibrous Scaffold using Quantitative NanoMechanical Mapping Mode of Atomic Force Microscope, J. Basic Appl. Biol. Int. 2 (2015) 2394-5820.

[43]H.G. Abdelhady, S. Allen, M.C. Davies, C.J. Roberts, S.J.B. Tendler, P.M. Williams, Atomic force microscopy studies of generation 4 poly(amidoamine) (PAMAM) dendrimers on functionalized surfaces, Surf. Sci. 558 (2004) 99-110. https://doi.org/10.1016/j.susc.2004.03.049.

[44]F. Awaja, M. Gilbert, G. Kelly, B. Fox, P.J. Pigram, Adhesion of polymers, Prog. Polym. Sci. 34 (2009) 948-968. https://doi.org/10.1016/j.progpolymsci.2009.04.007.

[45]C.C. Bueno, P.S. Garcia, C. Steffens, D.K. Deda, F. de Lima Leite, 5 - Nanosensors, in: A.L. Da Róz, M. Ferreira, F. de Lima Leite, O.N. Oliveira (Eds.), Nanosci. Its Appl., 
William Andrew Publishing, 2017: pp. 121-153. https://doi.org/10.1016/B978-0-32349780-0.00005-3.

[46]E. Peterson, C. Joseph, H. Peterson, R. Bouwman, S. Tang, J. Cannon, K. Sinniah, S.K. Choi, Measuring the Adhesion Forces for the Multivalent Binding of VancomycinConjugated Dendrimer to Bacterial Cell-Wall Peptide, Langmuir. 34 (2018) 7135-7146. https://doi.org/10.1021/acs.langmuir.8b01137.

[47]N. Tomczak, G.J. Vancso, Elasticity of Single Poly(amido amine) Dendrimers, Macromol. Rapid Commun. 28 (2007) 1640-1644. https://doi.org/10.1002/marc.200700202.

[48]V.V. Tsukruk, S. Singamaneni, Scanning Probe Microscopy of Soft Matter: Fundamentals and Practices, John Wiley \& Sons, 2012.

[49]J. Hu, Y. Cheng, Y. Ma, Q. Wu, T. Xu, Host-Guest Chemistry and Physicochemical Properties of the Dendrimer-Mycophenolic Acid Complex, J. Phys. Chem. B. 113 (2009) 64-74. https://doi.org/10.1021/jp8078919.

[50]L. Zhao, Q. Wu, Y. Cheng, J. Zhang, J. Wu, T. Xu, High-Throughput Screening of Dendrimer-Binding Drugs, J. Am. Chem. Soc. 132 (2010) 13182-13184. https://doi.org/10.1021/ja106128u.

[51]K. Yang, Y. Cheng, X. Feng, J. Zhang, Q. Wu, T. Xu, Insights into the Interactions between Dendrimers and Multiple Surfactants: 5. Formation of Miscellaneous Mixed Micelles Revealed by a Combination of ${ }^{1} \mathrm{H}$ NMR, Diffusion, and NOE Analysis, J. Phys. Chem. B. 114 (2010) 7265-7273. https://doi.org/10.1021/jp1026493.

[52]Y. Cheng, Shao, Zhang, Hu, Zhang, Zhang, Comparison of generation 3 polyamidoamine dendrimer and generation 4 polypropylenimine dendrimer on drug loading, complex structure, release behavior, and cytotoxicity, Int. J. Nanomedicine. (2011) 3361. https://doi.org/10.2147/IJN.S27028.

[53]R. Müller, C. Laschober, W.W. Szymanski, G. Allmaier, Determination of Molecular Weight, Particle Size, and Density of High Number Generation PAMAM Dendrimers Using MALDI-TOF-MS and nES-GEMMA, Macromolecules. 40 (2007) 5599-5605. https://doi.org/10.1021/ma062599e.

[54]A. Enciso, B. Neun, J. Rodriguez, A. Ranjan, M. Dobrovolskaia, E. Simanek, Nanoparticle Effects on Human Platelets in Vitro: A Comparison between PAMAM and $\begin{array}{lllll}\text { Triazine } & \text { Dendrimers, } & \text { Molecules. } & 21 & \text { (2016) }\end{array}$ https://doi.org/10.3390/molecules21040428. 
[55]M.M. Ulaszewska, M.D. Hernando, A.U. Moreno, A.V. García, E. García Calvo, A.R.

770

771

772

773

774

775

776

777

778

779

780

781

782

783

784
Fernández-Alba, Identification and quantification of poly(amidoamine) PAMAM dendrimers of generations 0 to 3 by liquid chromatography/hybrid quadrupole time-offlight mass spectrometry in aqueous medium, Rapid Commun. Mass Spectrom. RCM. 27 (2013) 747-762. https://doi.org/10.1002/rcm.6498.

[56]D. Shcharbin, A. Janaszewska, B. Klajnert-Maculewicz, B. Ziemba, V. Dzmitruk, I. Halets, S. Loznikova, N. Shcharbina, K. Milowska, M. Ionov, A. Shakhbazau, M. Bryszewska, How to study dendrimers and dendriplexes III. Biodistribution, pharmacokinetics and toxicity in vivo, J. Controlled Release. 181 (2014) 40-52. https://doi.org/10.1016/j.jconrel.2014.02.021.

[57]A. Uclés, M.J. Martínez Bueno, M.M. Ulaszewska, M.D. Hernando, C. Ferrer, A.R. Fernández-Alba, Quantitative determination of poly(amidoamine) dendrimers in urine by liquid chromatography/electrospray ionization hybrid quadrupole linear ion trap mass spectrometry, Rapid Commun. Mass Spectrom. RCM. 27 (2013) 2519-2529. https://doi.org/10.1002/rcm.6713. 


\section{Tables}

Table 1. Average particle size, polydispersity index, and zeta potential of OS-PAMAM and

PAMAM-G6 through 4 months at $5 \pm 3^{\circ} \mathrm{C}$.

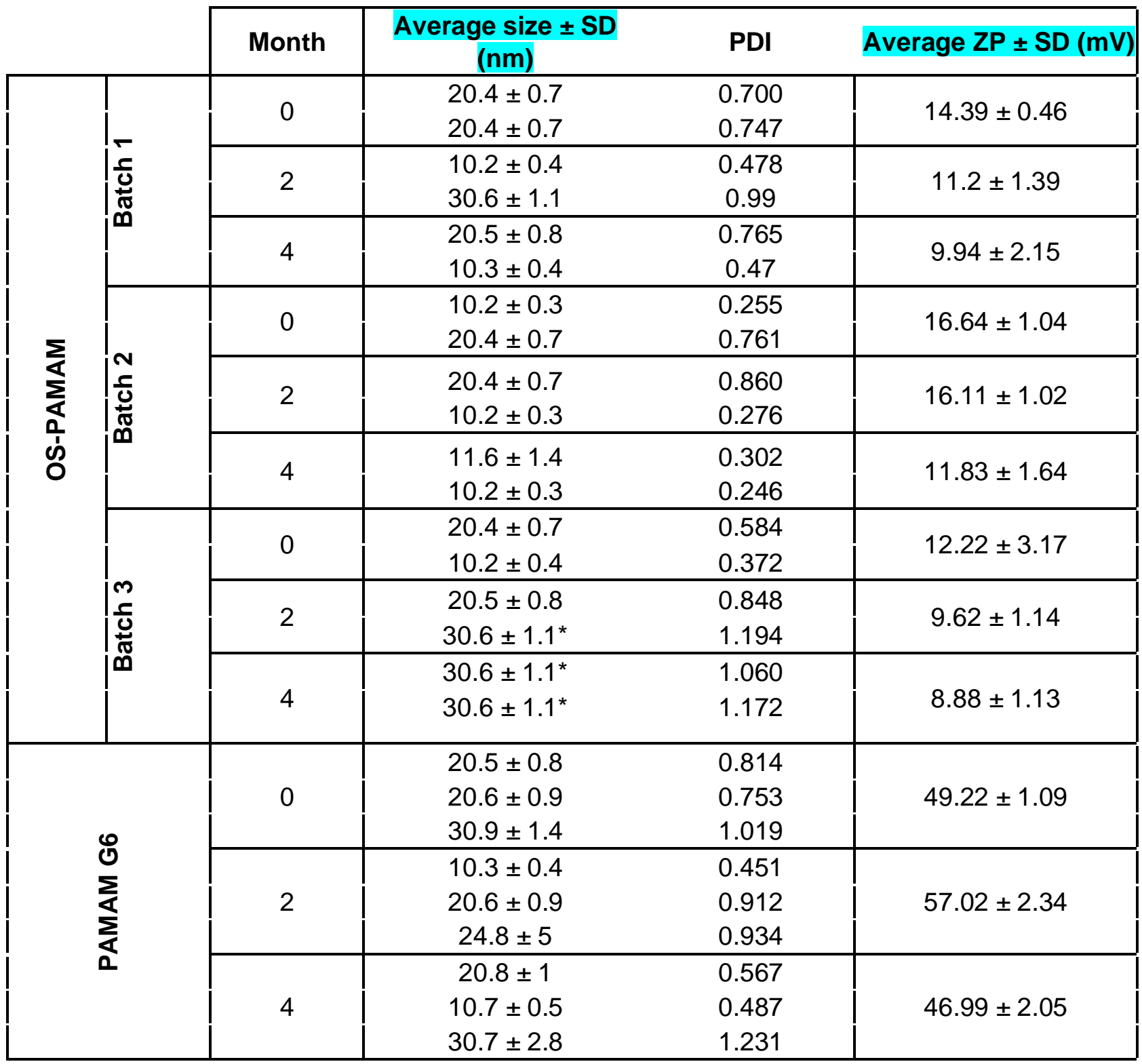


795 Table 2. Orthogonality between polydispersity indexes (PDI, $\mathbf{\Xi}$ in figure $8 \mathrm{~A}$ ) and average 796 molecular weights (Da) of OS-PAMAM and PAMAM-G6 obtained in the present study with 797 Dynamic Light Scattering (DLS), Size Exclusion Chromatography (SEC) and Diffusion Ordered 798 (DOSY) NMR spectroscopy. Zeta Potential (ZP) is highlighted for both dendrimers. PDIs with 799 DLS herein reported were constructed by the arithmetical average and standard deviations of 800 the full set of both OS-PAMAM batches and PAMAM-G6 dendrimers through four months 801 reported in Table 1. The SEC-PDI of OS-PAMAM here reported comes from the analysis of 802 three batches.

803

\begin{tabular}{|c|c|c|c|c|c|c|}
\hline & \multicolumn{2}{|c|}{ DLS } & \multicolumn{2}{c|}{ SEC } & \multicolumn{2}{c|}{ DOSY } \\
& OS-PAMAM & PAMAM-G6 & OS-PAMAM PAMAM-G6 & OS-PAMAM PAMAM-G6 \\
\hline \multirow{2}{*}{ PDI } & $\begin{array}{c}0.671 \pm 0.31 \\
Z P \sim 14 \mathrm{mV}\end{array}$ & $\begin{array}{c}0.796 \pm 0.24 \\
Z P \sim 20 \mathrm{mV}\end{array}$ & $1.05 \pm 0.039$ & 3.16 & $1.005 \pm 0.05$ & $1.02 \pm 0.05$ \\
\hline MW & & 8500 & 83000 & 5056.47 & 55711.86 \\
\hline
\end{tabular}

804

805

806

807

808

809

810

811

812 


\section{Captions}

815 Figure 1. UV-Vis spectra of OS-PAMAM and PAMAM-G6. (A) Spectrum with characteristic 816 signals of three independent production batches of OS-PAMAM vs PAMAM G6. (B) The signals 817 corresponding to tertiary amides from 280 to $285 \mathrm{~nm}$ were taken as a reference value to monitor 818 batches' integrity and control PAMAM-G6 through time until four months at $5 \pm 3{ }^{\circ} \mathrm{C}$.(C) 819 Structure of generation 3 (G3) PAMAM dendrimer with amino groups at the periphery. The ethylenediamine core is circumvented by a dashed line, reproduced from Abbasi et al., 2014 [4].

Figure 2. FT-IR spectra of compared dendrimers. The signals of OS-PAMAM (black) at 3270 $\mathrm{cm}^{-1} / 1637 \mathrm{~cm}^{-1}$ and $3089 \mathrm{~cm}^{-1} / 1546 \mathrm{~cm}^{-1}$ correspond with the signals at $3275 \mathrm{~cm}^{-1} / 1637$ $\mathrm{cm}^{-1}$ of primary and $3087 \mathrm{~cm}^{-1} / 1547 \mathrm{~cm}^{-1}$ of secondary amides, respectively, from PAMAMG6 dendrimers (blue).

Figure 3. Size and polydispersity index (PDI) relation and potential zeta profile. The average hydrodynamic sizes as a function of the polydispersity index (PDI) of (A) OS-PAMAM $(n=18)$ and $(B)$ PAMAM G6 $(n=9)$ were determined. Representative zeta potential profile of (C) OS-PAMAM and (D) PAMAM G6. All data were obtained under the same analytic method conditions by dynamic light scattering (DLS).

Figure 4. Molecular weight (MW) between OS-PAMAM batches and PAMAM G6 by SEC. (A) Chromatogram of PAMAM-G6 dendrimer at $215 \mathrm{~nm}$ (black line) was compared to three independent batches of OS-PAMAM (green, blue, and orange line) at $\mathrm{pH} \mathrm{6.8.} \mathrm{MW} \mathrm{was}$ determined by linear regression of the maximum value in absorbance units (A.U.) based on the MW standard curve (data not showed). (B) Second derivative analysis of OS-PAMAM batches and PAMAM G6.

Figure 5. Size and morphological analysis of dendrimers by AFM. Heightmap of (A) PAMAM-G6 and (B) OS-PAMAM, both average sizes were analyzed through the maximum vertical radius $(R z)$ of cross-sections $(n=50)$. (C) The dotted line represents a close-up of the 3D image of the typical shape of OS-PAMAM and PAMAM-G6 dendrimers.

841 Figure 6. AFM adhesion force profiles between dendrimers. (A) 2D (left) and 3D (right) 842 topographical imaging of OS-PAMAM and PAMAM-G6 was performed through automatic 
analysis of curves using NanoScope Analysis Software monitoring force-versus-distance curves. (B) Average adhesion force values $(\mathrm{nN})$ of cross-sections from OS-PAMAM and PAMAM-G6. Data represent mean \pm S.D. $(n=50)$.

Figure 7. AFM elasticity modulus differences between OS-PAMAM and PAMAM G6. (A) The elasticity in DMT modulus maps of OS-PAMAM and PAMAM-G6 was performed through automatic analysis of curves using NanoScope Analysis Software comparing hard (slide) and elastic (dendrimers) surfaces. (B) Average elasticity values (MPa) of cross-sections from OSPAMAM and PAMAM-G6. Data represent mean $\pm S D(n=50),{ }^{*} P<0.01$ vs OS-PAMAM.

Figure 8. Standard ${ }^{1} \mathrm{H}$-one dimensional direct-polarization NMR spectra. Spectra of PAMAM-G6 (blue) and OS-PAMAM (red) with any apodization prior to Fourier Transform (A and $C$ ) and proton spectra weighted with a Lorentz to Gauss line sharpening apodization function ( $B$ and $D$ ) used to reveal sample heterogeneity.

Figure 9. Solution-state PFG-STE-H2O(presat)-DOSY of PAMAM-G6 and OS-PAMAM. For obtaining average diffusion coefficient distributions (DCD), weighted average molecular weights and polydispersity indexes. (A) PFG-STE-H2O (presat)-DOSY-F2 assigned- NMR spectra of PAMAM batches: Commercial G6 PAMAM (Black) and synthetized OS-PAMAM (gray). Below each logarithmic diffusion coefficient average (Ln DCav, highlighted with a horizontal dotted line) are reported the weight-average molecular weights $(\dot{\mathrm{Mw}})$ and the polydispersity indexes (PDI) per case. (B) Relative molecular weights $[M w=f(D C D, D C a v)]$ of each chemical-shift assigned resonance obtained from DCD and DCav relationships represented as histograms and expressed in Daltons, are compared with reported molecular weights of PAMAM dendrimers by generation (G1: 1430 Da; G2: 3256 Da; G3: 6909 Da; G4: 14215 Da; G5: 28826 Da and G6: 58048 Da, from http://www.dendritech.com/pamam.html).

Figure 10. LC/ESI-QTOF-MS in high energy spectrum of precursor and fragmentation ions of OS-PAMAM compared with commercial PAMAM-G6. The precursor ions shared between OS-PAMAM and PAMAM-G6 were indicated according to the theoretical values reported for PAMAM G0-G3 and were colored in black. The precursor ions $(\mathrm{m} / \mathrm{z})$ of OS-PAMAM dendrimers dominated by a common fragmentation pattern based on the retro-Michael 
mechanism were colored in blue. The table below summarizes the theoretical $\mathrm{m} / \mathrm{z}$ values of the 874 OS-PAMAM and the PAMAM-G6 compared. All OS-PAMAM values present an error of less 875 than $10 \mathrm{ppm}$ concerning the theoretical one. ${ }^{*}$ The theoretical values were obtained from 876 Ulaszewska et al., 2013 [55].

877 Figure S1. Ideal (A) and non-ideal (B) amidation of PAMAM G0 dendrimers.

878 Figure S2. Molecular weight (MW) of OS-PAMAM and PAMAM G6 by SEC. Chromatogram 879 at $215 \mathrm{~nm}$ of PAMAM-G6 dendrimer (blue line) compared to 3 independent batches of OS880 PAMAM (green, red, and purple line) at $\mathrm{pH} 2.5$ condition before five months stored at 881 commercial conditions (methanol at $5 \pm 3^{\circ} \mathrm{C}$ ). MW of OS-PAMAM batches was calculated 882 considering PAMAM-G6 as reference.

883 Figure S3. LC/ESI-QTOF-MS in high energy spectrum of precursor and fragmentation 884 ions of OS-PAMAM batches. The precursor ions shared between OS-PAMAM batch 1 (L1) 885 and batch 2 (L2) indicate the reproducibility of the synthesis method between batches. 